TRANSACTIONS OF THE

AMERICAN MATHEMATICAL SOCIETY

Volume 351, Number 9, Pages 3533-3548

$\mathrm{S}$ 0002-9947(99)02039-5

Article electronically published on May 21, 1999

\title{
SPECTRAL GAP ESTIMATES ON COMPACT MANIFOLDS
}

\author{
KEVIN ODEN, CHIUNG-JUE SUNG, AND JIAPING WANG
}

\begin{abstract}
For a compact Riemannian manifold with boundary, its mass gap is the difference between the first and second smallest Dirichlet eigenvalues. In this paper, taking a variational approach, we obtain an explicit lower bound estimate of the mass gap for any compact manifold in terms of geometric quantities.
\end{abstract}

\section{INTRODUCTION}

Let $(M, g)$ be a compact Riemannian manifold with non-empty boundary, $\partial M$. The Laplace-Beltrami operator (Laplacian) acting on functions on $M$ is defined by

$$
\Delta(f)=\frac{1}{\sqrt{G}} \frac{\partial}{\partial_{i}}\left(\sqrt{G} g^{i j} \frac{\partial f}{\partial_{j}}\right),
$$

where the metric on $M$ is given by $d s^{2}=g_{i j} d x^{i} \otimes d x^{j},\left(g^{i j}\right)=\left(g_{i j}\right)^{-1}$ and $G=$ $\operatorname{det}\left(g_{i j}\right)$.

Eigenvalues of the Laplacian under Dirichlet boundary conditions are constants $l_{i}$ which satisfy

$$
\left\{\begin{array}{c}
\Delta u_{i}+l_{i} u_{i}=0, \quad x \in M \\
u_{i}(x)=0, \quad x \in \partial M
\end{array}\right.
$$

for some nonzero eigenfunctions $u_{i}$. It is well known that the spectrum of the Dirichlet problem satisfies

$$
\operatorname{Spec}_{D}(M)=\left\{0<l_{1}<l_{2} \leq l_{3} \cdots \rightarrow \infty\right\},
$$

so that the gap $l_{2}-l_{1}$, sometimes referred to as the "mass gap", is nontrivial.

In $[\mathrm{S}-\mathrm{W}-\mathrm{Y}-\mathrm{Y}]$ the mass gap is estimated by analyzing the function $\phi=\frac{u_{2}}{u_{1}}$. They show that

$$
\Delta \phi+2 \nabla \log u_{1} \cdot \nabla \log \phi+\left(l_{2}-l_{1}\right) \phi=0 .
$$

Therefore, $l_{2}-l_{1}$ appears as an eigenvalue of a certain partial differential operator. Moreover, since it is easy to see that $\phi$ is smooth on $M$ and satisfies $\frac{\partial \phi}{\partial \nu}=0, l_{2}-l_{1}$ is a Neumann eigenvalue of a certain partial differential operator. Thus, the gradient estimate techniques of $\mathrm{Li}$ and Yau $[\mathrm{L}-\mathrm{Y}]$ can be employed to show that

$$
l_{2}-l_{1} \geq \frac{\pi}{4 D^{2}}
$$

where $M$ is a convex Euclidean domain and $D$ is the diameter of $M$. The assumption of convexity seems to be crucial, however, to the argument since the Hessian

Received by the editors August 22, 1995 and, in revised form, February 13, 1997.

1991 Mathematics Subject Classification. Primary 58C40.

(C)1999 American Mathematical Society 
of $\log \left(u_{1}\right)$ appears in the gradient estimate calculation. To control this term a logconcavity result of Brascamp and Lieb [B-L] is used. The result fails for non-convex domains and manifolds.

The first author and Cheng [C-O] were able to estimate the mass gap on Euclidean domains satisfying a rolling R-ball condition,

$$
l_{2}-l_{1} \geq C(R, H, V, n),
$$

where the second fundamental form of the boundary, $\Pi$, satisfies $\Pi \geq-H(H \geq 0)$, $V$ is the volume of $M$ and $n$ is the dimension. This was done by introducing a weighted Cheeger's constant

$$
h_{u}=\inf \frac{\int_{H} u}{\min \left\{\int_{M_{1}} u, \int_{M_{2}} u\right\}}
$$

where the infimum is taken over all hypersurfaces $H$ with $M \backslash H=M_{1} \cup M_{2}$ and $\partial M_{1} \cap \partial M_{2}=H$. They then show that

$$
l_{2}-l_{1} \geq \frac{1}{4} h_{u}^{2}
$$

and give a lower bound for $h_{u}$ in terms of $R, H, V$ and $n$ (see [C-O] for details).

In this paper, we show (Theorem 1.3)

$$
l_{2}-l_{1} \geq C(K, H, R, D)
$$

on a compact Riemannian manifold with nonempty boundary satisfying a rolling $\mathrm{R}$-ball condition. In this formulation, $-K$ bounds the Ricci curvature from below, $H$ is a positive, semi-definite matrix which bounds the second fundamental form of $\partial M, R$ is determined by the rolling ball condition and $D$ is the diameter of $M$.

As in $[\mathrm{C}-\mathrm{O}]$, the key to the estimation technique is finding a lower bound for a $\phi^{2}$ weighted Rayleigh-Ritz quotient on $M$, with some appropriately chosen $\phi$ (see Proposition 1.1, below). For suitable $\phi$, for example the first Dirichlet eigenfunction, and bounded Euclidean domains satisfying rolling R-ball conditions, one can control the global behavior of the weighting function in terms of the distance function to the boundary - a Harnack-type inequality. Replacing the weighting function with the distance function allows one to use simple calculus to estimate the Rayleigh-Ritz quotient on $M$. Though these techniques do not carry over directly to the manifold setting, further study of the argument in [C-O] reveals that the essential elements of the estimation process are a Harnack inequality for the weighting function of the form $\phi(x) \leq C \phi(y)$ for all $x, y \in M$ satisfying $0<d(x, \partial M)<2 d(y, \partial M)$, where $C$ is constant and the use of a weak NeumannPoincaré inequality on the manifold (see assumptions 1-4, below). The rolling ball condition leads to the Harnack inequality by controlling the growth of $\phi$ near the boundary of $M$ in terms of the boundary geometry $(\mathrm{H})$ and the global geometry $(\mathrm{K})$. This naturally leads to an estimate of the constant, $C$, in terms of a possibly very small $R$, dependent on the smallest focal length as detailed in the remark following the proof of Theorem 1.3 and derived explicitly in $\S 2$.

The organization of this paper is as follows: In $\S 1$ we introduce notation and basic definitions and then prove the main theorem. In $\S 2$, which can be read independently, we prove a comparison result for the first Dirichlet eigenfunction. This verifies assumption (4), which is necesssary for the main theorem. 
It is our pleasure to thank Professors S. Y. Cheng, Peter Li, Rick Schoen and S.-T. Yau for their interest and encouragement. We would also like to thank the referee for pointing out many typos and shortcomings in the original draft.

1.

Let $l_{1}$ and $l_{2}$ be the first and second Dirichlet eigenvalues of $M$. We start with the following proposition which was established by S. Y. Cheng and the first author in $[\mathrm{C}-\mathrm{O}]$.

Proposition 1.1 (Cheng \& Oden).

$$
l_{2}-l_{1}=\inf _{f \in C^{1}(\bar{M})} \frac{\int_{M}|\nabla f|^{2} u^{2} d x}{\inf _{k \in \mathbb{R}} \int_{M}|f-k|^{2} u^{2} d x}
$$

where $u$ is the positive eigenfunction corresponding to $l_{1}$, i.e. $\Delta u-l_{1} u=0$ and $u>0$ on $M$.

Proof.

$$
\begin{aligned}
& \inf _{f \in C^{1}(\bar{M})} \frac{\int_{M}|\nabla f|^{2} u^{2}}{\inf _{k \in \mathbb{R}} \int_{M}|f-k|^{2} u^{2}} \\
& =\inf _{\substack{f \in C^{1}(\bar{M}) \\
\int_{M} f u^{2}=0}} \frac{\int_{M}|\nabla f|^{2} u^{2}}{\int_{M} f^{2} u^{2}} \\
& =\inf _{\substack{f \in C^{1}(\bar{M}) \\
\int_{M} f u^{2}=0}} \frac{\int_{M}\left(|\nabla(f u)|^{2}-f^{2}|\nabla u|^{2}-2 f u\langle\nabla f, \nabla u\rangle\right.}{\int_{M}(f u)^{2}} \\
& =\inf _{\substack{f \in C^{1}(\bar{M}) \\
f_{M} f u^{2}=0}} \frac{\int_{M}|\nabla(f u)|^{2}-\int_{M} f^{2}|\nabla u|^{2}+\frac{1}{2} \int_{M} f^{2} \Delta\left(u^{2}\right)}{\int_{M}(f u)^{2}} \\
& =\inf _{\substack{f \in C^{1}(\bar{M}) \\
\int_{M} f u^{2}=0}} \frac{\int_{M}|\nabla(f u)|^{2}-l_{1} \int_{M} f^{2} u^{2}}{\int_{M}(f u)^{2}} \\
& =\inf _{\substack{f \in C^{1}(\bar{M}) \\
f_{M} f u^{2}=0}} \frac{\int_{M}|\nabla(f u)|^{2}}{\int_{M}(f u)^{2}}-l_{1} \\
& \geq \inf _{\substack{g \in C_{0}^{1}(\bar{M}) \\
\int_{M} g u=0}} \frac{\int_{M}|\nabla g|^{2}}{\int_{M} g^{2}}-l_{1} \geq l_{2}-l_{1} .
\end{aligned}
$$

On the other hand, let $v$ be an eigenfunction of $l_{2}$. Then $f_{0}=\frac{v}{u} \in C^{\infty}(\bar{M})$ as shown in $[\mathrm{S}-\mathrm{W}-\mathrm{Y}-\mathrm{Y}]$ and

$$
l_{2}-l_{1}=\frac{\int_{M}\left|\nabla f_{0}\right|^{2} u^{2}}{\inf _{k \in \mathbb{R}} \int_{M}\left|f_{0}-k\right|^{2} u^{2}} \geq \inf _{f \in C^{1}(\bar{M})} \frac{|\nabla f|^{2} u^{2}}{\inf _{k \in \mathbb{R}} \int_{M}|f-k|^{2} u^{2}} .
$$

Thus, the proposition is proved.

In the following, we are going to use the variational characterization of $l_{2}-l_{1}$ to give a lower bound estimate. We consider more generally the problem of estimating

$$
\inf _{f \in C^{1}(\bar{M})} \frac{\int_{M}|\nabla f|^{2} \varphi^{2} d x}{\inf _{k \in \mathbb{R}} \int_{M}|f-k|^{2} \varphi^{2} d x}
$$


where $\varphi(x)$ is a given function on $M$ and $\varphi>0$ on $M$. We assume

1. $M$ satisfies volume doubling property, i.e. $\exists C_{1}>0$ s.t. for any ball $B_{x}(2 r) \subset$ $M$ we have $\frac{\left|B_{x}(2 r)\right|}{\left|B_{x}(r)\right|} \leq C_{1}$.

2. $M$ satisfies interior rolling R-ball condition. i.e. for all $x \in \partial M, \exists B_{p}(R) \subset M$ s.t. $B_{p}(R) \cap \partial M=\{x\}$.

3. $M$ satisfies a weak Neumann-Poincaré inequality on balls, i.e. for any ball $B_{x}(r) \subset M, B_{x}(r) \cap \partial M=\varnothing$, we have

$$
\inf _{k \in \mathbb{R}} \int_{B_{x}\left(\frac{r}{2}\right)}|f-k|^{2} d x \leq C_{2} r^{2} \int_{B_{x}(r)}|\nabla f|^{2} d x
$$

for all $f \in C^{1}(\bar{M})$, where $C_{2}$ is a constant independent of $x$ and $r$.

4. $\exists C_{3}>0$ s.t. $\varphi(x) \leq C_{3} \phi(y)$ for all $x, y \in M$ with $0<d(x, \partial M) \leq 2 d(y, \partial M)$.

Theorem 1.2. Under assumptions 1, 2, 3, and 4, we have

$$
\inf _{f \in C^{1}(\bar{M})} \frac{\int_{M}|\nabla f|^{2} \varphi^{2} d x}{\inf _{k \in \mathbb{R}} \int_{M}|f-k|^{2} \varphi^{2} d x} \geq C\left(C_{1}, C_{2}, C_{3}, R, \mu, D\right),
$$

where $\mu$ is the first nonzero Neumann eigenvalue of $M_{\frac{R}{2}}=\{x \in M: d(x, \partial M) \geq$ $\left.\frac{R}{2}\right\}$ and $D=\operatorname{diam}(M)$, the diameter of $M$.

Our proof of the theorem is adapted from $[\mathrm{J}]$ and $[\mathrm{SC}-\mathrm{S}]$.

Proof. We begin with the following claim.

Claim 1. There exists a collection of balls $\mathcal{F}=\left\{B_{x_{i}}\left(r_{i}\right): i \in I\right\}$ s.t.

(i) $\bigcup_{i \in I} B_{x_{i}}\left(2 r_{i}\right)=M$ and $B_{x_{i}}\left(r_{i}\right) \cap B_{x_{j}}\left(r_{j}\right)=\varnothing$ for any $i \neq j, i, j \in I$,

(ii) $d\left(B_{x_{i}}\left(r_{i}\right), \partial M\right)=10^{3} r_{i}$,

(iii) $\#\left\{B_{x_{i}}\left(r_{i}\right) \in \mathcal{F}: \bigcap B_{x_{i}}\left(10 r_{i}\right) \neq \varnothing\right\} \leq C\left(C_{1}\right)$.

In fact, let $B_{1}$ be a ball in $M$ of largest radius $r_{1}$ satisfying $d\left(B_{1}, \partial M\right)=10^{3} r_{1}$. Let $B_{2}$ be a ball in $M$ of largest radius $r_{2}$ satisfying $d\left(B_{2}, \partial M\right)=10^{3} r_{2}$ and $B_{2} \cap B_{1}=\varnothing$. Keep choosing balls in this way to obtain $\mathcal{F}$. We check that $\mathcal{F}$ satisfies (i), (ii) and (iii). Let $x \in M$. Then $\exists r>0$ s.t. $d\left(B_{x}(r), \partial M\right)=10^{3} r$. We may assume $B_{x}(r) \notin \mathcal{F}$. It is clear then $\exists B \in \mathcal{F}$ s.t. the radius of $B$ satisfies $r(B) \geq r$ and $B \cap B_{x}(r) \neq \varnothing$.

Write $B=B_{x_{i}}\left(r_{i}\right)$. Then for $y \in B_{x_{i}}\left(r_{i}\right) \cap B_{x}(r)$,

$$
d\left(x, x_{i}\right) \leq d(x, y)+d\left(y, x_{i}\right) \leq r+r_{i} \leq 2 r_{i} .
$$

Thus $x \in B_{x_{i}}\left(2 r_{i}\right)$ and $M=\bigcup_{i \in I} B_{x_{i}}\left(2 r_{i}\right)$. So (i) follows. (ii) is trivially true by the construction. To check (iii), suppose $\bigcap_{i \in J} B_{x_{i}}\left(10 r_{i}\right) \neq \varnothing$. Let $y \in \bigcap_{i \in J} B_{x_{i}}\left(10 r_{i}\right)$. Then $d\left(x_{i}, y\right) \leq 10 r_{i}$. Thus for any $x \in B_{x_{i}}\left(r_{i}\right), d(x, y) \leq d\left(x, x_{i}\right)+d\left(x_{i}, y\right) \leq 11 r_{i}$. Hence $\bigcup_{i \in J} B_{x_{i}}\left(r_{i}\right) \subset B_{y}(20 r)$, where $r=\max \left\{r_{i}\right\}=r_{i_{0}}$. On the other hand,

$$
d\left(x_{i_{0}}, x_{j}\right) \leq d\left(x_{i}, y\right)+d\left(y, x_{j}\right) \leq 10 r_{i_{0}}+10 r_{j} \leq 20 r .
$$

So

$$
\begin{aligned}
d\left(x_{j}, \partial M\right) & \geq d\left(x_{i_{0}}, \partial M\right)-d\left(x_{i_{0}}, x_{j}\right) \\
& \geq d\left(B_{x_{i_{0}}}\left(r_{i_{0}}\right), \partial M\right)-20 r \\
& \geq 10^{3} r_{i_{0}}-20 r>101 r .
\end{aligned}
$$


Hence $d\left(B_{x_{j}}\left(r_{j}\right), \partial M\right)>100 r$ and $r_{j}>10^{-1} r$. In conclusion,

$$
\bigcup_{i \in J} B_{x_{i}}\left(r_{i}\right) \subset B_{y}(20 r) \subset B_{x_{j}}(30 r) \subset B_{x_{j}}\left(300 r_{j}\right)
$$

Therefore

$$
\sum_{i \in J}\left|B_{x_{i}}\left(r_{i}\right)\right| \leq\left|B_{y}(20 r)\right| \leq \frac{\sum_{j \in J}\left|B_{x_{j}}\left(300 r_{j}\right)\right|}{\#\left\{B_{x_{i}}\left(r_{i}\right): \bigcap B_{x_{i}}\left(10 r_{i}\right) \neq \varnothing\right\}} .
$$

Hence

$$
\#\left\{B_{x_{i}}\left(r_{i}\right): \bigcap B_{x_{i}}\left(10 r_{i}\right) \neq \varnothing\right\} \leq \frac{\sum_{j \in J}\left|B_{x_{j}}\left(300 r_{j}\right)\right|}{\sum_{j \in J}\left|B_{x_{j}}\left(r_{j}\right)\right|} \leq C\left(C_{1}\right)
$$

and (iii) follows.

Now let $M_{R}=\{x \in M ; d(x, \partial M) \geq R\}$. Let $\mathcal{L}=\left\{B_{i} \in \mathcal{F}: x_{i} \notin M_{R}\right\}$. For $B_{i} \in \mathcal{L}$, let $y_{i} \in \partial M$ s.t. $d\left(x_{i}, y_{i}\right)=d\left(x_{i}, \partial M\right)$. By the interior rolling R-ball condition, $\exists B_{q_{i}}(R)$ s.t. $B_{q_{i}}(R) \cap \partial M=y_{i}$. Then clearly $d\left(q_{i}, y_{i}\right)=d\left(q_{i}, \partial M\right)=R$. Let $\overline{q_{i} y_{i}}$ be a minimal geodesic. Then $\overline{q_{i} y_{i}} \perp \partial M$. In particular, this implies such a minimal geodesic is unique up to a reparametrization. Also, let $\overline{x_{i} y_{i}}$ be a minimal geodesic realizing the distance between $x_{i}$ and $\partial M$. Then $\overline{x_{i} y_{i}} \perp \partial M$. This forces $x_{i} \in \overline{q_{i} y_{i}}$. We denote by $l_{i}$ the segment $\overline{q_{i} x_{i}}$ of $\overline{q_{i} y_{i}}$. We then define, for $B_{i} \in \mathcal{L}$, $\mathcal{F}\left(B_{i}\right)=\left\{A \in \mathcal{F}: 2 A \cap l_{i} \neq \varnothing\right\}$. Let $\mathcal{H}=\left\{A \in \mathcal{F}: A \in \mathcal{F}\left(B_{i}\right)\right.$ for some $\left.B_{i} \in \mathcal{L}\right\}$. We have the following claim.

Claim 2. (i) $A \in \mathcal{F}(B)$ implies $r(A) \geq 10^{-1} r(B)$.

(ii) For any $A \in \mathcal{H}$, let

$$
A(\mathcal{L})=\{B \in \mathcal{L}: A \in \mathcal{F}(B)\} .
$$

Then

$$
r(A)^{2}|A|^{-1} \sum_{B \in A(\mathcal{L})} \# \mathcal{F}(B)|B| \leq C\left(C_{1}\right) R^{2} .
$$

We first check (i). For $A \in \mathcal{F}(B), 2 A \cap l_{B} \neq \varnothing$. Let $y \in 2 A \cap l_{B}$ and $x$ be the center of the ball $B$. Then

$$
\begin{aligned}
d(A, \partial M) & \geq d(y, \partial M)-d(A, y) \\
& \geq d(x, \partial M)-d(A, y) \\
& \geq 10^{3} r(B)-d(A, y) \\
& \geq 10^{3} r(B)-2 r(A) .
\end{aligned}
$$

Thus $10^{3} r(A) \geq 10^{3} r(B)-2 r(A)$ and $r(A) \geq 10^{-1} r(B)$.

To check (ii), we show

(a) $\#\left\{A \in \mathcal{F}(B): r \leq r(A) \leq \frac{11}{10} r\right\} \leq C\left(C_{1}\right)$ for any $r>0$, where $C$ is independent of $r$.

(b) $\# \mathcal{F}(B) \leq C \ln \frac{C R}{r(B)}, C=C\left(C_{1}\right)$.

(c) $\exists \varepsilon=\varepsilon\left(C_{1}\right)>0$ s.t.

$$
\sum_{B \in A(\mathcal{L}), r \leq r(B) \leq 2 r}|B| \leq C|A|\left(\frac{r}{r(A)}\right)^{\varepsilon} \text { for } A \in \mathcal{H}
$$

where $r>0$ is arbitrary, $C=C\left(C_{1}\right)$ constant. 
For (a), suppose $A_{1}, A_{2} \in\left\{A \in \mathcal{F}(B): r \leq r(A) \leq \frac{11}{10} r\right\}$ and $y_{1} \in 2 A_{1} \cap l_{B}, y_{2} \in$ $2 A_{2} \cap l_{B}$. Let $A_{1}=B_{\xi_{1}}\left(r_{1}\right)$ and $A_{2}=B_{\xi_{2}}\left(r_{2}\right)$. Since for $i=1,2$,

$$
d\left(y_{i}, \partial M\right) \geq d\left(\xi_{i}, \partial M\right)-d\left(\xi_{i}, y_{i}\right) \geq 10^{3} r_{i}-2 r_{i}
$$

and

$$
d\left(y_{i}, \partial M\right) \leq d\left(\xi_{i}, \partial M\right)+d\left(\xi_{i}, y_{i}\right) \leq 10^{3} r_{i}+3 r_{i}
$$

we have

$$
\begin{aligned}
d\left(y_{1}, y_{2}\right) & \leq\left|d\left(y_{1}, \partial M\right)-d\left(y_{2}, \partial M\right)\right| \\
& \leq\left(10^{3}+3\right) \frac{11}{10} r-\left(10^{3}-2\right) r \\
& \leq 15 r
\end{aligned}
$$

Now it is clear that

$$
A_{2} \subset B_{y_{1}}(20 r) \text { and } d\left(y_{1}, \partial M\right) \geq 998 r .
$$

Therefore

$$
\bigcup\left\{A \in \mathcal{F}(B): r \leq r(A) \leq \frac{11}{10} r\right\} \subset B_{y_{1}}(20 r) .
$$

Using the volume doubling property, we conclude

$$
\#\left\{A \in \mathcal{F}(B): r \leq r(A) \leq \frac{11}{10} r\right\} \leq C\left(C_{1}\right) .
$$

Now (b) follows from (a). In fact, $A \in \mathcal{F}(B)$ implies that $10^{-1} r(B) \leq r(A) \leq R$. Therefore

$$
\begin{aligned}
\# \mathcal{F}(B) \leq & \#\left\{A \in \mathcal{F}(B): 10^{-1} r(B) \leq r(A) \leq \frac{11}{10} 10^{-1} r(B)\right\} \\
& +\quad \#\left\{A \in \mathcal{F}(B): \frac{11}{10} 10^{-1} r(B) \leq r(A) \leq\left(\frac{11}{10}\right)^{2} 10^{-1} r(B)\right\} \\
& +\ldots+\#\left\{A \in \mathcal{F}(B):\left(\frac{11}{10}\right)^{k} 10^{-1} r(B) \leq r(A) \leq\left(\frac{11}{10}\right)^{k+1} 10^{-1} r(B)\right\}
\end{aligned}
$$

where $k$ satisfies $\left(\frac{11}{10}\right)^{k} 10^{-1} r(B) \leq R \leq\left(\frac{11}{10}\right)^{k+1} 10^{-1} r(B)$. From (a) we know $\# \mathcal{F}(B) \leq C k$. But $k \leq C \ln \frac{C R}{r(B)}$. Thus $\# \mathcal{F}(B) \leq C \ln \frac{c R}{r(B)}$.

Now we come to (c). First, note that for $B \in A(\mathcal{L}), r(B)<10 r(A)$ by (i) of Claim 2. So $\frac{r}{r(A)}<10$. Choose $\eta_{0} \in \partial M$ s.t. $d\left(\eta_{0}, A\right)=d(A, \partial M)=10^{3} r(A)$. Let $s=10^{10} r(A)$. For $\delta>0$, denote

$$
R(\delta)=\left\{\eta \in B\left(\eta_{0}, s+2 \delta s\right): d(\eta, \partial M)<\delta s\right\} .
$$

Then for $B \in A(\mathcal{L})$ and $r(B) \leq 2 r$, we have $B \subset R\left(\frac{r}{r(A)}\right)$. In fact, let $B=$ $B_{x}(r(B))$. Then

$$
d(x, \partial M) \leq d(B, \partial M)+r(B) \leq 10^{3} r(B)+2 r \leq 10^{4} r .
$$


So $B \subset\left\{\eta: d(\eta, \partial M)<10^{10} r\right\}$. To show $B \subset B\left(\eta_{0}, s+2 \delta s\right), \delta=\frac{r}{r(A)}$, note that $A \in \mathcal{F}(B)$. Therefore, $\exists y \in \overline{x q} \cap 2 A$. Hence

$$
d(x, y) \leq d(y, \partial M) \leq 3 r(A)+d(A, \partial M) \leq\left(10^{3}+3\right) r(A) .
$$

So

$$
d\left(x, \eta_{0}\right) \leq d(x, y)+d\left(y, \eta_{0}\right) \leq 2\left(10^{3}+3\right) r(A)
$$

and for $z \in B_{x}(r(B))$,

$$
d\left(z, \eta_{0}\right) \leq d(z, x)+d\left(x, \eta_{0}\right)<10^{10} r(A)+2 r .
$$

Therefore $B_{x}(r(B)) \subseteq R\left(\frac{r}{r(A)}\right)$. On the other hand, by the volume doubling property, we have $|A| \approx\left|B\left(\eta_{0}, 2 s\right)\right|$. Thus, (c) follows if we can show

$$
\left.|R(\delta)| \leq C \delta^{\varepsilon} \mid B(\eta), 2 s\right) \mid, \quad 0<\delta<10 .
$$

We first show that $\exists \sigma\left(C_{1}\right)>0$ s.t.

$$
(1+\sigma)\left|R\left(\frac{1}{4} \delta\right)\right| \leq|R(\delta)| \text { for } 0<\delta<10^{10} .
$$

Choose a maximal set of points $\xi_{1}, \ldots, \xi_{N} \in \partial M$ s.t. $B\left(\xi_{i}, \delta s\right) \cap B\left(\eta_{0}, s\right) \neq \varnothing$ and $\left\{B\left(\xi_{i}, \delta s\right) ; i=1, \ldots, N\right\}$ are pairwise disjoint. It is then easy to check that

$$
R\left(\frac{1}{4} \delta\right) \subset \bigcup_{j=1}^{N} B\left(\xi_{j}, 10 \delta s\right) .
$$

Also, $\exists B\left(\eta_{j}, \frac{\delta s}{2}\right) \subset \bar{M} \backslash \partial M$ s.t. $d\left(\eta_{j}, \xi_{j}\right) \leq \frac{\delta s}{2}$. In fact, since $A \in \mathcal{H}, A \in \mathcal{F}(B)$ for some $B \in \mathcal{L}$. Thus, $d(A, \partial M) \leq r(A)+R$ and $r(A) \leq 10^{-2} R$. By the interior rolling R-ball condition, $\exists q_{j}$ s.t. $B_{q_{j}}(R) \cap \partial M=\left\{\xi_{j}\right\}$. Now choose $\eta_{j} \in \overline{q_{j} \xi_{j}}$ s.t. $d\left(\eta_{j}, \xi_{j}\right)=\frac{\delta s}{2} \leq \frac{r(A)}{2}<10^{-2} R$. Then

$$
B\left(\eta_{j}, \frac{\delta s}{2}\right) \subset B_{q_{j}}(R) \subset \bar{M} \backslash \partial M \text { and } d\left(\eta_{j}, \xi_{j}\right)=\frac{\delta s}{2} .
$$

Now we have

$$
B\left(\eta_{j}, \frac{\delta s}{4}\right) \subset R(\delta) \quad \text { and } \quad B\left(\eta_{j}, \frac{\delta s}{4}\right) \cap R\left(\frac{1}{4} \delta\right)=\varnothing .
$$

Moreover, $\exists \sigma>0$ s.t. $\left|B\left(\eta_{j}, \frac{\delta s}{4}\right)\right|>\sigma\left|B\left(\xi_{j}, 10 \delta s\right)\right|$. Therefore,

$$
\sum_{j=1}^{N}\left|B\left(\eta_{j}, \frac{\delta s}{4}\right)\right|>\sigma \sum_{j=1}^{N}\left|B\left(\xi_{j}, 10 \delta s\right)\right| \geq \sigma\left|R\left(\frac{1}{4} \delta\right)\right| .
$$

Consequently,

$$
|R(\delta)| \geq\left|R\left(\frac{1}{4} \delta\right)\right|+\left|\bigcup_{j=1}^{N} B\left(\eta_{j}, \frac{\delta s}{4}\right)\right|>(1+\sigma)\left|R\left(\frac{1}{4} \delta\right)\right|
$$

as the balls $B\left(\eta_{j}, \frac{\delta s}{4}\right)$ are mutually disjoint. 
For any $\delta>0$, write $\delta \cong\left(\frac{1}{4}\right)^{k} \delta_{0}$, where $\delta_{0}$ fixed. Then

$$
|R(\delta)| \leq\left(\frac{1}{1+\sigma}\right)^{k}\left|R\left(\delta_{0}\right)\right| \leq C \delta^{\varepsilon}\left|B\left(\eta_{0}, 2 s\right)\right| .
$$

Thus (c) follows.

Now (ii) of Claim 2 can be checked. In fact,

$$
\begin{aligned}
& r(A)^{2}|A|^{-1} \sum_{B \in A(\mathcal{L})} \# \mathcal{F}(B)|B| \\
\leq & r(A)^{2}|A|^{-1} \sum_{B \in A(\mathcal{L})} C \ln \frac{C R}{r(B)}|B| \\
\leq & r(A)^{2}|A|^{-1} \sum_{k=-\infty}^{0} \sum_{2^{k} \in A(\mathcal{L})} C \ln \frac{C R}{r(B)}|B| \\
\leq & r(A)^{2}|A|^{-1} \sum_{k=-\infty}^{0} C \ln \frac{C R}{2^{k} r(A)}|A|\left(\frac{2^{k} r(A)}{r(A)}\right)^{\varepsilon} \\
\leq & r(A)^{2} \sum_{k=-\infty}^{0}\left[\left(C \ln \frac{C R}{r(A)}\right) 2^{k \varepsilon}-(C \ln 2) k 2^{k \varepsilon}\right] \\
\leq & r(A)^{2}\left[C \ln \frac{C R}{r(A)}+C\right] \\
\leq & C R^{2} \text { as } r(A) \leq C R .
\end{aligned}
$$

With those two claims, we can now finish the proof of the theorem.

For $B \in \mathcal{L}$, let $A_{1}, \ldots, A_{l}$ be the elements in $\mathcal{F}(B)$ s.t. $A_{1}=B, q \in 2 A_{l}$. Let

$$
\begin{aligned}
f_{B}^{\prime}= & \int_{B} \frac{f \varphi^{2}}{\int_{B} \varphi^{2}}, f_{0}^{\prime}=\frac{\int_{M_{R / 2}} f \varphi^{2}}{\int_{M_{R / 2}} \varphi^{2}} \text {. Then } \\
& \int_{4 B}\left|f-f_{0}^{\prime}\right|^{2} \varphi^{2} \\
\leq & 2 \int_{4 B}\left|f-f_{4 B}^{\prime}+\sum_{i=1}^{l-2}\left(f_{4 A_{i}}^{\prime}-f_{4 A_{i+1}}^{\prime}\right)\right|^{2} \varphi^{2}+2 \int_{4 B} \varphi^{2}\left|f_{l-1}^{\prime}-f_{0}^{\prime}\right|^{2} \\
\leq & 2 l\left[\int_{4 B}\left|f-f_{4 B}^{\prime}\right|^{2} \varphi^{2}+\int_{4 B} \varphi^{2} \sum_{i=1}^{l-2}\left|f_{4 A_{i}}^{\prime}-f_{4 A_{i+1}}^{\prime}\right|^{2}\right]+2 \int_{4 B} \varphi^{2}\left|f_{l-1}^{\prime}-f_{0}^{\prime}\right|^{2} .
\end{aligned}
$$

Since

$$
\begin{aligned}
& \int_{4 A_{i} \cap 4 A_{i+1}} \varphi^{2}\left|f_{4 A_{i}}^{\prime}-f_{4 A_{i+1}}^{\prime}\right|^{2} \\
\leq & 2\left[\int_{4 A_{i}}\left|f-f_{4 A_{i}}^{\prime}\right|^{2} \varphi^{2}+\int_{4 A_{i+1}}\left|f-f_{4 A_{i+1}}^{\prime}\right|^{2} \varphi^{2}\right],
\end{aligned}
$$


and

$$
\begin{aligned}
& \int_{4 B} \varphi^{2}\left|f_{l-1}^{\prime}-f_{0}^{\prime}\right|^{2} \\
\leq & \frac{\int_{4 B} \varphi^{2}}{\int_{4 A_{l-1} \cap M_{R / 2}} \varphi^{2}}\left(\int_{4 A_{l-1} \cap M_{R / 2}} \varphi^{2}\left|f_{l-1}^{\prime}-f_{0}^{\prime}\right|^{2}\right) \\
\leq & 2 \frac{\int_{4 B} \varphi^{2}}{\int_{4 A_{l-1} \cap M_{R / 2}} \varphi^{2}}\left(\int_{4 A_{l-1} \cap M_{R / 2}}\left(\left|f-f_{l-1}^{\prime}\right|^{2}+\left|f-f_{0}^{\prime}\right|^{2}\right) \varphi^{2}\right) \\
\leq & 2 \frac{\int_{4 B} \varphi^{2}}{\int_{4 A_{l-1} \cap M_{R / 2}} \varphi^{2}}\left(\int_{4 A_{l-1}}\left|f-f_{l-1}^{\prime}\right|^{2} \varphi^{2}+\int_{M_{R / 2}}\left|f-f_{0}^{\prime}\right|^{2} \varphi^{2}\right),
\end{aligned}
$$

we conclude

$$
\begin{aligned}
& \int_{4 B}\left|f-f_{0}^{\prime}\right|^{2} \varphi^{2} \\
\leq & 2 l\left[\int_{4 B}\left|f-f_{4 B}^{\prime}\right|^{2} \varphi^{2}+\sum_{i=1}^{l-2} \frac{\int_{4 B} \varphi^{2}}{\int_{4 A_{i} \cap 4 A_{i+1}} \varphi^{2}} \int_{4 A_{i} \cap 4 A_{i+1}} \varphi^{2}\left|f_{4 A_{i}}^{\prime}-f_{4 A_{i+1}}^{\prime}\right|^{2}\right] \\
& +4 \frac{\int_{4 B} \varphi^{2}}{\int_{4 A_{l-1} \cap M_{R / 2}} \varphi^{2}}\left(\int_{4 A_{l-1}}\left|f-f_{l-1}^{\prime}\right|^{2} \varphi^{2}+\int_{M_{R / 2}}\left|f-f_{0}^{\prime}\right|^{2} \varphi^{2}\right) \\
\leq \quad & 4 l\left[\int_{4 B}\left|f-f_{4 B}^{\prime}\right|^{2} \varphi^{2}\right. \\
& \left.+\sum_{i=1}^{l-2} \frac{\int_{4 B} \varphi^{2}}{\int_{4 A_{i} \cap 4 A_{i+1}} \varphi^{2}}\left(\int_{4 A_{i}}\left|f-f_{4 A_{i}}^{\prime}\right|^{2} \varphi^{2}+\int_{4 A_{i+1}}\left|f-f_{4 A_{i+1}}^{\prime}\right|^{2} \varphi^{2}\right)\right] \\
& +4 \frac{\int_{4 B} \varphi^{2}}{\int_{4 A_{l-1} \cap M_{R / 2}} \varphi^{2}}\left(\int_{4 A_{l-1}}\left|f-f_{l-1}^{\prime}\right|^{2} \varphi^{2}+\int_{M_{R / 2}}\left|f-f_{0}^{\prime}\right|^{2} \varphi^{2}\right) .
\end{aligned}
$$

From assumption (4) on $\varphi$, it is easy to see that

$$
\frac{\int_{4 B} \varphi^{2}}{\int_{4 A_{i} \cap 4 A_{i+1}} \varphi^{2}} \leq C\left(C_{3}\right) \frac{|B|}{\left|4 A_{i} \cap 4 A_{i+1}\right|} \leq C\left(C_{1}, C_{3}\right) \frac{|B|}{\left|A_{i}\right|}
$$

and

$$
\frac{\int_{4 B} \varphi^{2}}{\int_{4 A_{l-1} \cap M_{R / 2}} \varphi^{2}} \leq C\left(C_{1}, C_{3}\right) \frac{|B|}{\left|A_{l-1}\right|} .
$$

On each $A_{i}$, we also have $\varphi(x) \leq C_{2} \varphi(y)$ for all $x, y \in A_{i}$. Therefore by assumption $(3)$,

$$
\int_{4 A_{i}}\left|f-f_{i}^{\prime}\right|^{2} \varphi^{2} \leq C_{2} r^{2}\left(A_{i}\right) \int_{4 A_{i}}|\nabla f|^{2} \varphi^{2} .
$$

On $M_{R / 2}, \varphi(x) \leq C_{4}\left(C_{3}, R, D\right) \varphi(y)$ for $x, y \in M_{R / 2}$, where $D$ is the diameter of $M$. Thus

$$
\int_{M_{R / 2}}\left|f-f_{0}^{\prime}\right|^{2} \varphi^{2} \leq \frac{C_{4}}{\mu} \int_{M_{R / 2}}|\nabla f|^{2} \varphi^{2}
$$


In conclusion, we have

$$
\begin{aligned}
\int_{4 B}\left|f-f_{0}^{\prime}\right|^{2} \varphi^{2} \leq & C \# \mathcal{F}(B)|B| \sum_{A_{i} \in \mathcal{F}(B)} r\left(A_{i}\right)^{2}\left|A_{i}\right|^{-1} \int_{4 A_{i}}|\nabla f|^{2} \varphi^{2} \\
& +\frac{C_{4}|B|}{\mu\left|A_{l}\right|} \int_{M_{R / 2}}|\nabla f|^{2} \varphi^{2} .
\end{aligned}
$$

Using assumption (1), it is easy to see $\left|A_{l}\right| \geq C\left(C_{1}, D, R\right)|M|$. Thus

$$
\begin{aligned}
\int_{4 B}\left|f-f_{0}^{\prime}\right|^{2} \varphi^{2} \leq & C \# \mathcal{F}(B)|B| \sum_{A_{i} \in \mathcal{F}(B)} r\left(A_{i}\right)^{2}\left|A_{i}\right|^{-1} \int_{4 A_{i}}|\nabla f|^{2} \varphi^{2} \\
& +\frac{C_{5}|B|}{\mu|M|} \int_{M_{R / 2}}|\nabla f|^{2} \varphi^{2} .
\end{aligned}
$$

Summing over all $B \in \mathcal{L}$, we have

$$
\begin{aligned}
& \int_{M \backslash M_{R}}\left|f-f_{0}^{\prime}\right|^{2} \varphi^{2} \\
\leq & \sum_{B \in \mathcal{L}} \int_{4 B}\left|f-f_{0}^{\prime}\right|^{2} \varphi^{2} \\
\leq & C \sum_{B \in \mathcal{L}} \# \mathcal{F}(B)|B| \sum_{A \in \mathcal{F}(B)} r(A)^{2}|A|^{-1} \int_{4 A}|\nabla f|^{2} \varphi^{2}+C_{5} \frac{\left|M \backslash M_{R}\right|}{\mu|M|} \int_{M_{R / 2}}|\nabla f|^{2} \varphi^{2} \\
\leq & C \sum_{A \in \mathcal{H}} r(A)^{2}|A|^{-1} \sum_{B \in A(\mathcal{L})}|B| \# \mathcal{F}(B) \int_{4 A}|\nabla f|^{2} \varphi^{2}+\frac{C_{5}}{\mu} \int_{M_{R / 2}}|\nabla f|^{2} \varphi^{2} \\
\leq & C R^{2} \int_{M}|\nabla f|^{2} \varphi^{2}+\frac{C_{5}}{\mu} \int_{M_{R / 2}}|\nabla f|^{2} \varphi^{2},
\end{aligned}
$$

where we have used (ii) of Claim 2. Thus

$$
\begin{aligned}
\int_{M}\left|f-f_{0}^{\prime}\right|^{2} \varphi^{2} & \leq \int_{M \backslash M_{R}}\left|f-f_{0}^{\prime}\right|^{2} \varphi^{2}+\int_{M_{R / 2}}\left|f-f_{0}^{\prime}\right|^{2} \varphi^{2} \\
& \leq\left(C R^{2}+\frac{C_{5}+1}{\mu}\right) \int_{M}|\nabla f|^{2} \varphi^{2} .
\end{aligned}
$$

Therefore

$$
\frac{\int_{M}|\nabla f|^{2} \varphi^{2}}{\inf _{k \in \mathbb{R}} \int_{M}|f-k|^{2} \varphi^{2}} \geq \frac{1}{C R^{2}+\frac{C_{6}}{\mu}}
$$

where $C_{6}=C\left(C_{1}, C_{2}, C_{3}, R, D\right)$ and $C=C\left(C_{1}, C_{2}, C_{3}\right)$. The theorem is proved.

Remark. Notice that the assumptions (1), (2) and (3) are stable under quasiisometry on $(M, g)$.

In order to apply Theorem 1.1 to estimate $l_{2}-l_{1}$, we need to verify assumptions (1), (2) and (3) for $(M, g)$ and (4) for $u$. (4) is verified in $\S 2$. To verify assumptions (1), (2) and (3) we assume now the following for $(M, g)$.

(5) $\operatorname{Ric}_{M} \geq-K$ on $M$ for some constant $K \geq 0$. 
(6) The second fundamental form of $\partial M$ w.r.t. the outward unit normal satisfies $\Pi \geq-H$, where $H \geq 0$.

Theorem 1.3. Suppose $(M, g)$ satisfies assumptions (2), (5) and (6). Then $l_{2}-$ $l_{1} \geq C(K, H, R, D)$, where $R$ is chosen to be "small". (See Remark.)

Proof. It has been shown in [W] that $M$ satisfies (1) with $C_{1}=C(K, R)$ by arranging $R$ "small". $M$ satisfies (3) by fact $\operatorname{Ric}_{M} \geq-K$ and $C_{2}=C(K, D)$ (see [SC-S]). To estimate $\mu$ from below, we use the result in $[\mathrm{C}]$.

Note first that $M_{R / 2}$ satisfies interior r-rolling ball condition with $r \geq \frac{R}{4}$. In fact, $\forall x \in \partial M_{R / 2}, \exists y \in \partial M$ s.t. $d(x, y)=d(x, \partial M)$. Since $M$ satisfies interior R-rolling ball condition, $\exists B_{p}(R) \subset M$ s.t. $B_{p}(R) \cap \partial M=\{y\}$. Now let $\overline{p y}$ be the minimal geodesic. Let $q \in \overline{p y}$ s.t. $d(p, q)=\overline{p q}=\frac{1}{4} R$. Then it can be easily checked that $B_{q}\left(\frac{1}{4} R\right) \subset M_{R / 2}$ and $B_{q}\left(\frac{1}{4} R\right) \cap \partial M_{R / 2}=\{x\}$.

Next, we want to estimate the second fundamental form of $\partial M_{R / 2}$ from below. Let $f(x)=d(x, \partial M)$. Then $M_{R / 2}=\left\{f(x) \geq \frac{R}{2}\right\}$ and $\partial M_{R / 2}=\left\{f(x)=\frac{R}{2}\right\}$. Since $|\nabla f|=1$ on $M \backslash M_{R}$, it is easy to see that $\Pi_{\partial M_{R / 2}}=H e s s i a n(f)$ on $M$. By the index comparison theorem in $[\mathrm{H}-\mathrm{K}]$ or $[\mathrm{Wr}]$, we have, by choosing $R$ small,

$$
\operatorname{Hessian}(f) \geq-C(R, H) \text {. }
$$

Thus, $\Pi_{\partial M_{R / 2}} \geq-C(R, H)$. By $[\mathrm{C}]$, we conclude that

$$
\mu \geq C(R, H, K, D)
$$

In conclusion, we have

$$
l_{2}-l_{1} \geq C(R, D, K, H)
$$

where $R$ is chosen to be "small".

Remark. The number $R$ is chosen to satisfy the following:

(a) $\sqrt{K_{R}} \tan \left(R \sqrt{K_{R}}\right) \leq \frac{H}{2}+\frac{1}{2}$,

(b) $H \tan \left(R \sqrt{K_{R}}\right) \leq \frac{1}{2} \sqrt{K_{R}}$, where $K_{R}$ is the upper bound of the sectional curvature of $M$ on the set $M \backslash M_{R}$. Clearly, if $M$ is a Euclidean domain, then $K_{R}=0$ and $R$ can be taken as in (2).

2 .

In this section we will verify property (4) for the first eigenfunction given Dirichlet conditions on a manifold $M$ with $\partial M \neq \emptyset$.

In order to do this we will need bounds on $\Delta d(x, \partial M)$ for $x$ "close" to $\partial M$. To this end we recall the notation from $\S 1$ and set

$$
M_{\delta}=\{x \in M \mid d(x, \partial M)>\delta\} .
$$

We define a map $\Psi: \partial M \times[0, \infty) \longrightarrow M$ by setting $\Psi(y, \rho)=\exp \left(\rho N_{y}\right)$ where $N_{y}$ is the inward pointing unit normal at $y \in \partial M$ and recall the following standard facts about $\Psi$ :

1. If $\partial M$ satisfies an interior rolling R-ball condition, then there exists $\delta>0$ such that $\Psi: \partial M \times[0, \delta) \longrightarrow M$ is $C^{2}$.

2. For the $\delta$ of $(1), d(x, \partial M)=d(x, y)=\rho$, and $\rho(x)$ is $C^{2}$ for $x \in M \backslash M_{\delta}$. 
3. If $d V$ is the volume element on $M$ and $\Phi$ is the volume element on $\partial M$, then $\Psi^{*}(d V)=\mathcal{H}(y, \rho) \Phi \wedge d \rho$ and $\Delta \rho(x)=\Delta d(x, \partial M)=\frac{\mathcal{H}^{\prime}}{\mathcal{H}}$ on $M \backslash M_{\delta}$.

Many proofs of properties (1), (2) and (3) can be found in the literature. A proof of property (3) can be found in [G-H-L]. Proofs of (1),(2) and (3) can also be found in $[\mathrm{O}]$. In the next lemma we shall bound $\Delta d(x, \partial M)$ as well as get an estimate on $\delta$.

Lemma 2.1. Let $\rho(x)=d(x, \partial M)$. Suppose we have constants $b \geq a>0, b \geq 1$ such that $-a^{2} \leq \operatorname{Sec}(M) \leq b^{2}$. Then $\rho$ is $C^{k}$ on $M \backslash M_{\delta}$, if $\partial M$ is $C^{k}$ and

$$
\sum_{i=1}^{n-1} \frac{-b \sin b \rho-k_{i}(y) \cos b \rho}{\cos b \rho-\frac{k_{i}(y)}{b} \sin b \rho} \leq \Delta \rho \leq \sum_{i=1}^{n-1} \frac{-a \sinh a \rho-k_{i}(y) \cosh a \rho}{\cos h a \rho-\frac{k_{i}(y)}{a} \sinh a \rho}
$$

where $k_{i}(y)$ are the principal curvatures on $\partial M$ at the unique point $y \in \partial M$ such that $\rho(x)=d(x, y)$ and

$$
\delta=\frac{1}{4(n-1)(b+H+1 / R)} .
$$

Proof. Let $x \in M$ with $\rho(x)=d(x, \partial M)=d(x, y)$ with $y \in \partial M$. Suppose $k_{i}$ are the principal curvatures at $y$. At least locally, on the space form of constant curvature $b^{2}$ we may immerse a hypersurface with curvatures $k_{i}$. To derive the first inequality, we consider a hypersurface $\bar{H}$ immersed in $S^{n}(1 / b)$, the $n$-sphere of radius $1 / b$. The second inequality is derived by replacing $S^{n}(1 / b)$ with the hyperbolic space $H^{n}(1 / a)$ and repeating the argument. Let $D \subseteq \bar{H}$ be an open set and define, as above, $\bar{\Psi}: D \times[0, \infty) \rightarrow S^{n}(1 / b)$ by

$$
\bar{\Psi}(y, \bar{\rho})=\exp \bar{\rho} N_{y}
$$

where $N_{y}$ is the inward pointing unit normal at $y$. Since geodesics on $S^{n}(1 / b)$ starting at a point $y$ and having initial direction $N_{y}$ have the form

$$
\cos b \bar{\rho} y+\frac{1}{b} \sin b \bar{\rho} N_{y}
$$

we may write

$$
\bar{\Psi}(y, \bar{\rho})=\cos b \bar{\rho} y+\frac{1}{b} \sin b \bar{\rho} N_{y} .
$$

On $\bar{H}$ there exist $n-1$ orthonormal principal vectors $E_{i}$. We extend $E_{i}$ by parallel translation to vector fields $E_{i}(\bar{\rho})$ along the normal geodesic $\gamma(\bar{\rho})=\bar{\Psi}(y, \bar{\rho})$. Let $\gamma_{i}(t)$ be geodesics on $\bar{H}$ such that $\gamma_{i}(0)=y$ and $\gamma_{i}^{\prime}(0)=E_{i}(0)$. Then

$$
\begin{aligned}
\frac{\partial \bar{\Psi}}{\partial \gamma_{i}}(y, \bar{\rho}) & =\cos b \bar{\rho} \gamma_{i}^{\prime}(0)+\frac{1}{b} \sin b \bar{\rho} \frac{\partial N_{\gamma_{i}(t)}}{\partial \gamma_{i}}(0) \\
& =\cos b \bar{\rho} E_{i}-\frac{k_{i}}{b} \sin b \bar{\rho} E_{i} \\
& =\left(\cos b \bar{\rho}-\frac{\overline{k_{i}}}{b} \sin b \bar{\rho}\right) E_{i}
\end{aligned}
$$

Since $\left(\cos b \bar{\rho}-\frac{k_{i}}{b} \sin b \bar{\rho}\right) E_{i}$ are variation vector fields, they are in fact Jacobi fields and describe the Jacobian, $J(\bar{\Psi})$, of $\bar{\Psi}$ (see for instance $[\mathrm{H}-\mathrm{K}]$ ), and $J(\bar{\Psi})$ satisfies

$$
\begin{aligned}
J(\bar{\Psi})(y, \bar{\rho}) & =\left(\cos b \bar{\rho}-\frac{k_{1}}{b} \sin b \bar{\rho}\right)\left(\cos b \bar{\rho}-\frac{\overline{k_{2}}}{b} \sin b \bar{\rho}\right) \cdots\left(\cos b \bar{\rho}-\frac{k_{n-1}}{b} \sin b \bar{\rho}\right) \\
& >0 .
\end{aligned}
$$


The last inequality follows from $\bar{\rho} \leq \delta$ so that

$$
\cot b \bar{\rho} \geq \frac{2 H}{b} .
$$

We have used the fact that $\theta \longrightarrow 0, \tan \theta$ is approximately $\theta$. Therefore, we may apply the inverse function thereom to find an open set $D^{\prime} \subseteq D$ where $y$ is a $C^{k-1}$ function of $x=\bar{\Psi}(y, \bar{\rho})$. However, the definition of $\bar{\Psi}$ implies $\nabla \bar{\rho}=N_{y}$ is a $C^{k-1}$ function of $y$. Therefore, $\bar{\rho}$ is $C^{k}$. The index estimates on $\overline{\mathcal{H}}$ and $\mathcal{H}$ in [H-K] imply $J(\bar{\Psi})(y, \bar{\rho})=\overline{\mathcal{H}}(y, \bar{\rho}) \leq \mathcal{H}(y, \rho)$ where $\bar{\rho}=\rho$ which implies $\rho$ is $C^{k}$ on $M \backslash M_{\delta}$. Applying property (2) we have

$$
\Delta \rho \geq \Delta \bar{\rho}=\sum_{i=1}^{n-1} \frac{-b \sin b \rho-k_{i}(y) \cos b \rho}{\cos b \rho-\frac{k_{i}(y)}{b} \sin b \rho},
$$

which completes the proof of the lemma.

Since $\rho \leq \delta$ from Lemma 2.1 we can draw the following conclusions:

$$
\begin{aligned}
\Delta \rho & \geq \sum_{i=1}^{n-1} \frac{-b \sin b \rho-k_{i}(y) \cos b \rho}{\cos b \rho-\frac{k_{i}(y)}{b} \sin b \rho} \\
& =\sum_{i=1}^{n-1} \frac{-b \tan b \rho-k_{i}(y)}{1-\frac{k_{i}(y)}{b} \tan b \rho} \\
& \geq \sum_{i=1}^{n-1}-2\left(b+k_{i}\right) \\
& \geq-2(n-1)(b+H+1 / R) . \\
\Delta \rho^{2} & =2 \rho \Delta \rho+2|\nabla \rho|^{2} \\
\geq & 2 \rho(-2(n-1)(b+H+1 / R))+2 \\
\geq & -1+2 \\
\geq & 1 .
\end{aligned}
$$

Now we can place $\sup _{M} u$ on a compact subset of $M$ :

Lemma 2.2. Suppose $u$ is the first eigenfunction on $M$ and $u\left(x_{0}\right)=\sup _{x \in M} u$. Then on $M \backslash M_{\delta}$

$$
u(x) \leq \frac{6 \mu}{\delta} \rho(x) .
$$

Furthermore,

$$
d\left(x_{0}, \partial M\right) \geq \frac{\delta}{6 \mu}
$$

where

$$
\mu=\frac{(n-1) a^{2}}{4}+\frac{4 \pi^{2}}{\delta^{2}}+\frac{(n-1)^{2} a^{2}}{4 \sinh ^{2}(a / 2)} .
$$


Proof. Without loss of generality, assume $\sup _{x \in M} u=1$. Set $\gamma_{1}=2 /(3 \delta)$. Then using Lemma 2.1 and conclusions (2.1) and (2.2) we have

$$
\begin{aligned}
\Delta\left(\rho-\gamma_{1} \rho^{2}\right) & \leq \sum_{i=1}^{n-1} \frac{-a \tanh a \rho-k_{i}}{1-\frac{k_{i}}{a} \tanh a \rho}-\gamma_{1} \\
& \leq 2(n-1)(a+H+1 / R)-\frac{1}{2 \delta} \\
& \leq \frac{1}{2 \delta}-\frac{2}{3 \delta} \\
& =-\frac{1}{6 \delta} .
\end{aligned}
$$

A result of Gage ([G]) tells us that $\lambda \leq \mu$, so by setting $\gamma_{2}=6 \mu / \delta$ we have

$$
\begin{aligned}
\Delta\left(u-\gamma_{2}\left(\rho-\gamma_{1} \rho^{2}\right)\right) & \geq-\lambda u+\gamma_{2} \frac{1}{6 \delta} \\
& \geq-\lambda u+\mu \\
& \geq 0 .
\end{aligned}
$$

On $\partial M_{\delta}$ we have

$$
\begin{aligned}
u-\gamma_{2}\left(\rho-\gamma_{1} \rho^{2}\right) & =u-\gamma_{2}\left(\delta-\gamma_{1} \delta^{2}\right) \\
& =u-\gamma_{2}\left(\delta-\frac{1}{2} \delta\right) \\
& =u-\frac{6 \mu}{\delta} \frac{1}{2} \delta \\
& \leq u-3 \mu \\
& \leq 0 .
\end{aligned}
$$

The last inequality follows since $\mu$ is clearly greater than 1 . Therefore, by the maximum principle we have

$$
d(x, \partial M)=\rho(x) \geq \frac{1}{\gamma_{2}} u(x) .
$$

To bound $u$ from below is more involved. Again the maximum principle will be used but it is necessary for us to bound $u$ from below on $\{\rho=\epsilon\}$ for some suitable $\epsilon$. This is done in the following proposition.

Proposition 2.3. Let $u$ be the positive eigenfunction of $M$ with respect to Dirichlet boundary conditions and normalized so that $\sup _{M} u=1$. Then, for $d(x, \partial M) \geq \frac{\delta}{6 \mu}$ we have

$$
u(x) \geq \exp \left(-\frac{24 \mu d_{M}}{\delta} \log \left(\frac{50(n-1)^{2}}{\delta}\left(\mu^{2}+a\right)\right)\right) .
$$

Proof. Proposition says that $\sup _{M_{\epsilon}} u=1$ with $\epsilon=\frac{6 \mu}{\delta}$. We will apply the Harnack inequality to bound $u$ from below on $M_{\epsilon}$. For any $x \in M_{\epsilon}, B_{\epsilon}(x) \subseteq M$. The gradient estimate in $[\mathrm{S}-\mathrm{Y}]$ says that

$$
\left|\frac{\nabla u}{u}\right| \leq 4 \frac{l_{1}^{2}}{\epsilon^{2}}+\frac{8(n-1)^{2}}{\epsilon^{2}}\left(\epsilon^{2}+a\right)
$$


on $B_{\epsilon / 2}(x)$. Therefore, on $B_{\epsilon / 2}(x)$,

$$
\sup _{B_{\epsilon / 2}(x)} u \leq \frac{l_{1}^{2}}{\epsilon}+\frac{8(n-1)^{2}}{\epsilon^{2}}(\epsilon+a) \inf _{B_{\epsilon / 2}(x)} u .
$$

Since $l_{1} \leq \mu$, we have

$$
\sup _{B_{\epsilon / 2}(x)} u \leq \frac{8(n-1)^{2}}{\epsilon}\left(\mu^{2}+a\right) \inf _{B_{\epsilon / 2}(x)} u .
$$

Since $\epsilon=\frac{6 \mu}{\delta} \leq \frac{R}{2}$ for $n \geq 2$, we see easily that $d_{M_{\epsilon}} \leq 2 d_{M}$. Applying (2.4) repeatedly on $M_{\epsilon}$ we derive that

$$
1=\sup _{M_{\epsilon}} u \leq\left(\frac{8(n-1)^{2}}{\epsilon}\left(\mu^{2}+a\right)\right)^{\frac{4 d_{M}}{\epsilon}} \inf _{M_{\epsilon}} u .
$$

Therefore,

$$
\inf _{M_{\epsilon}} u \geq \exp \left(-\frac{24 \mu d_{M}}{\delta} \log \left(\frac{50(n-1)^{2}}{\delta}\left(\mu^{2}+a\right)\right)\right) .
$$

This completes the proof of the proposition.

Now we can bound $u$ from below by some multiple of $\rho$.

Proposition 2.4. Let $u$ be the first positive eigenfunction of $M$ with respect to Dirichlet boundary conditions and normalized so that $\sup _{M} u=1$. For $x \in M$, $d(x, \partial M) \leq \frac{6 \mu}{\delta}$, we have

$$
u(x) \geq \rho(x) \frac{1}{4 \delta} \exp \left(-\frac{24 \mu d_{M}}{\delta} \log \left(\frac{50(n-1)^{2}}{\delta}\left(\mu^{2}+a\right)\right)\right) .
$$

Proof. Let $C(\delta)$ denote the right-hand side of (2.3).

Let $\alpha_{1}, \alpha_{2}$ be positive constants to be chosen later. Then on $T_{\delta}$,

$$
\begin{aligned}
\Delta\left(u-\alpha_{2}\left(\rho+\alpha_{1} \rho^{2}\right)\right) & =-l_{1} u-\alpha_{2}\left(\sum \frac{-b \sinh b \rho-k_{1} \cosh b \rho d}{\cos b \rho-\frac{k_{1}}{b} \sin b \rho}+\alpha_{1} \Delta \rho^{2}\right) \\
& \leq-l_{1} u-\alpha_{2}\left(-2(n-1)(b+H+1 / R)+\alpha_{1}\right) .
\end{aligned}
$$

Therefore, the right-hand side of (2.5) will be less than or equal to zero if $\alpha_{1} \geq$ $(n-1)(b+H+1 / R)$.

Set $\alpha_{1}=\frac{1}{\delta}$. Then, the infimum of $\left(u-\alpha_{2}\left(\rho+\alpha_{1} \rho^{2}\right)\right)$ on $M \backslash M_{\delta}$ occurs on the boundary of $M \backslash M_{\delta}$.

For $x \in M, d(x, \partial M)=\delta$, we have

$$
\begin{aligned}
u(x)-\alpha_{2}\left(\delta+\alpha_{1} \delta^{2}\right) & \geq C(\delta)-\alpha_{2} \delta\left(1+\frac{1}{\delta} \delta\right) \\
& \geq C(\delta)-2 \alpha_{2} \delta \\
& \geq 0
\end{aligned}
$$

if $\alpha_{2}=\frac{C(\delta)}{4 \delta}$.

So, with these choices of $\alpha_{1}, \alpha_{2}$ we then have on $M \backslash M_{\delta}$,

$$
u \geq \frac{C(\delta)}{4 \delta}\left(\rho+\frac{1}{\delta} \rho^{2}\right)
$$


and therefore

as stated in the proposition.

$$
u \geq \frac{C(\delta)}{4 \delta} \rho,
$$

Lemmas 2.1, 2.2 and Proposition 2.3 immediately give us property (4) of $\S 1$. That is,

Theorem 2.5. Let $M$ be a compact Riemannian manifold with $\partial M \neq \emptyset$, satisfying an interior rolling $\mathrm{R}$-ball condition and having sectional curvature $-a^{2} \leq \operatorname{Sec}(M) \leq$ $b^{2}$ with $b \geq a$. Let $\rho(x)=d(x, \partial M)$ and $u$ be the first eigenfunction satisfying Dirichlet conditions. Then on $M \backslash M_{\delta}$, with $\delta$ chosen as in Lemma 2.1, there are constants $C_{i}(a, b, H, R, D, n), i=1,2$, such that

$$
C_{1} \rho(x) \leq u(x) \leq C_{2} \rho(x) .
$$

Furthermore, there exists $C=C(a, b, H, R, D, n)>0$ such that $u(x) \leq C u(y)$ for all $x, y \in M$ with $0<d(x, \partial M) \leq 2 d(y, \partial M)$.

\section{REFERENCES}

[B-L] H. Brascamp, E. Lieb, J. Funct. Anal., 22 (1976) 366-389. MR 56:8774

[G] M. Gage, Indiana Univ. Math. J. 29 (1980) 897-912. MR 82b:58095

[G-H-L] S. Gallot, D. Hulin, J. Lafontaine, Riemannian Geometry, Springer-Verlag, New York (1990). MR 91j:53001

[J] D. Jerison, Duke Math. J. 53 (1986) no.2 503-523. MR 87i:35027

[C] R. Chen, Proc. AMS 108 (1990) no.4 961-970. MR 90g:58135

[L-Y] P. Li, S.T Yau, Proceedings of Symposia in Pure Math., Vol. 36 (1980), AMS, 205-230. MR 81i: 58050

[SC-S] L. Saloff-Coste and D. Stroock, J. Funct. Anal 98 (1991) 97-121. MR 92k:58264

[S-W-Y-Y] I.Singer, B.Wong, S. T. Yau and S. S. T. Yau, Ann. Scuola Norm. Sup. Pisa XXII (1985) 319-333. MR 87j:35280

[H-K] E. Heintze and H. Karcher, Ann. Scient. Ec. Norm. Sup. 11 (1978) 451-470. MR 80i:53026

[Wr] F. Warner, Trans. AMS 122 (1966) 341-356. MR 34:759

[C-O] S.Y. Cheng, K. Oden, Isoperimetric Inequalities and the Gap Between the First and Second Eigenvalues of an Euclidean domain, preprint.

[O] K. Oden, UCLA dissertation, 1994

[S-Y] R. Schoen, S.T. Yau, Differential Geometry, International Press (1995).

[W] J. Wang, Global heat kernel estimate, Pacific J. Math. 178 (1997) 377-398. MR 98g:58168

Department of Mathematics, Harvard University, Cambridge, Massachusetts 02138

Department of Mathematics, National Chung Cheng University, Taiwan

E-mail address: cjsung@math.ccu.edu.tw

Department of Mathematics, Stanford University, Stanford, California 94305 Current address: Department of Mathematics, Cornell University, Ithaca, New York 14853

E-mail address: jwang@math.cornell.edu 